\title{
ARTIGOS
}

\section{Características Demográficas da Terceira Idade na América Latina e no Brasil.}

\author{
Olga Collinet Heredia ${ }^{I}$
}

\begin{abstract}
Resumo
O objetivo do presente artigo é mostrar como o envelhecimento demográfico é um fenômeno mundial de grande impacto. Ainda que iniciado em momentos diferentes no mundo, dependendo do desenvolvimento econômico da região. Hoje ele se mostra igualmente desafiante em todas as latitudes. Aumenta a esperança de vida da população ao se produzir a queda da fecundidade e da mortalidade graças ao progresso econômico e as inovações médicas e aos avanços na saúde pública. Este fenômeno que vem intensificando-se desde a década de 60 apresenta também grandes desafios já que as nações devem começar a prepar-se para satisfazer as necessidades de seus cidadãos e de seus núcleos familiares.

Palavras-Chave: terceira idade; envelhecimento; demografia; América Latina; Brasil.
\end{abstract}

\begin{abstract}
The main objective of this article is to show how demographic agement is a worldwide phenomenon with great impact. Even if it has began in different moments in the world, it depends of the region economical development. Nowadays it shows like a challenge in all ways. Increasing the population hope of life we produce a reducement on the fertility and mortality due to the economical progress, medical inovations and advances on the public health. This phenomenon that is growing since the sixties also presents big challenges since that the nations have to begin to prepare themselves to satisfy the necessities of
\end{abstract}

* Mestre em Demografia (Universidade de Louvain), Professora e Pesquisadora da Universidade do Vale do Rio dos Sinos, São Leopoldo - RS. E-Mail: olga@bage.unisinos.br

Estud. interdiscip. envelhec., Porto Alegre, v.2, p.7-21, 1999. 
their citizens and their familiar nucleous.

Key-Words: third age; agement; demography; Latin America; Brazil.

\section{Introdução}

O processo de envelhecimento populacional vem manifestando-se em forma diferenciada no tempo, segundo desenvolvimento econômico, e a rapidez com que se apresenta. Este desenvolvimento influi no comportamento das variáveis demográficas que atuam no envelhecimento: mortalidade e natalidade.

Na Europa e América do Norte, com a Revolução Industrial, o fenômeno do envelhecimento apresentou-se há quase cem anos atrás. Nas outras regiões do mundo este processo começou a manifestar-se na década de sessenta e sua velocidade de expansão é extraordinária.

As estatísticas revelam que no início do século XXI existirão, no mundo, 600 milhões de pessoas maiores de 60 anos e perto de 15 milhões com mais de 85 anos.

Já em 1982, as Nações Unidas observaram que a situação populacional, com relação a envelhecimento, seria uma das preocupações mais urgentes nos próximos anos. Assim proclamaram até 2025 como a era do envelhecimento e determinaram como convenção uma idade de início deste fenômeno demográfico de acordo com o grau de desenvolvimento existente na região. Nos países denominados desenvolvidos essa idade é de 65 anos e nos subdesenvolvidos ou em vias de desenvolvimento de 60 anos.

\section{América Latina}

O continente Latino Americano está experimentando mudanças populacionais profundas que se manifestam na queda dos níveis de fecundidade e mortalidade infantil e com um baixo crescimento populacional. Mas existe grande heterogeneidade entre os países e o interior de cada um deles, pelo comportamento demográfico diferencial segundo setores sociais ou áreas de residências.

Apesar destes aspectos, observa-se, nos países do continente, um paulatino aumento na esperança de vida da população. Nos anos setenta esta 
chegava a quase 60 anos e, atualmente, situa-se perto dos 70, o que faz aumentar a participação da população idosa no total da população.

O presente trabalho tem por objetivo mostrar as causas que motivaram esta situação a nível do Continente Latino Americano e do Brasil.

\subsection{População Idosa e seu Crescimento}

Observando o crescimento da população de sessenta (60) anos e mais, em números absolutos no continente desde os anos de 1960, constata-se que esta população teve o seguinte comportamento:

Tabela 1 - Crescimento da População Latino Americana de 60 Anos e mais em Números Absolutos.

\begin{tabular}{l|l}
\hline ANOS & $\begin{array}{l}\text { POPULAÇÃO } \\
\text { (60 anos e mais })\end{array}$ \\
\hline 1960 & 12.185 .317 \\
1970 & 16.714 .562 \\
1980 & 22.672 .223 \\
1990 & 30.6866940 \\
2000 & 41.050 .337 \\
2010 & 56.364 .609 \\
2020 & 81.311483 \\
\hline
\end{tabular}

FONTE: CENTRO LATINOAMERICANO DE DEMOGRAFIA. Boletin Demográfico: América Latina, projecciones de población 1950-2050. Santiago de Chile, v.27, n. 54,1994.

Entre 1960 e 1990, esse grupo etário quase triplicou e as projeções para o século XXI mostram também um crescimento considerável até 2020.

Com relação ao crescimento, em número relativos da proporção de pessoas de 60 anos e mais no total da população, ou seja, o índice de envelhecimento, a América Latina experimentou o seguinte aumento: 
Tabela 2 - Índice de Envelhecimento em Percentuais.

\begin{tabular}{c|c}
\hline ANOS & $\begin{array}{c}\text { INDICE DE } \\
\text { ENVELHECIMENTO EM \% }\end{array}$ \\
\hline 1960 & 5,82 \\
1970 & 6,09 \\
1980 & 6,49 \\
1990 & 7,17 \\
2000 & 8,38 \\
2010 & 9,62 \\
2020 & 12,39 \\
\hline
\end{tabular}

FONTE: CENTRO LATINOAMERICANO DE DEMOGRAFIA. Boletin Demográfico: América Latina, projecciones de población 1950-2050. Santiago de Chile, v.27, n. 54,1994.

Este índice que determina a inserção da população idosa no total da população ao aumentar produz graves consequiências a nível econômico, social e político o que exige da parte das autoridades competentes, rápido posicionamento para encontrar soluções.

\section{2 Índices Demográficos Latino Americanos e sua Participação no Envelhecimento Populacional}

Ao olhar para esta mesma população idosa, agora classificada por sexo, observa-se que seu aumento em Números Absolutos e Relativos é o seguinte:

Tabela 3 - População Idosa em Números Absolutos e Relativos, Segundo o Sexo.

\begin{tabular}{l|r|l|r|r}
\hline ANOS & HOMENS & \multicolumn{1}{|c}{ \% } & MULHERES & \% \\
\hline 1960 & 5.796 .398 & 47,56 & 6.388 .909 & 52,44 \\
1970 & 7.889 .529 & 47,21 & 8.825 .033 & 52,79 \\
1980 & 10.599 .399 & 46,75 & 12.072 .828 & 53,25 \\
1990 & 14.252 .299 & 46,44 & 16.434 .638 & 53,56 \\
2000 & 18.968 .309 & 46,21 & 22.082 .029 & 53,79 \\
2010 & 25.967 .943 & 46,07 & 30.396 .663 & 53,93 \\
2020 & 37.296 .431 & 45,87 & 44.015 .054 & 54,13 \\
\hline
\end{tabular}

FONTE: CENTRO LATINOAMERICANO DE DEMOGRAFIA. Boletin Demográfico: América Latina, projecciones de población 1950-2050. Santiago de Chile, v.27, n. 54,1994. 
O crescimento da população feminina idosa é uma constatação tanto no final do século XX quanto nas projeções do próximo milênio para América Latina.

Esta situação pode ser observada quando se utiliza a relação de sexo, índice que demostra como diminui a proporção de homens com relação a cem (100) mulheres com o aumento da idade.

Tabela 4 - Percentual de Homens Idosos em Relação a Mulheres Idosas.

\begin{tabular}{c|c}
\hline ANOS & R. de Sexos.\% \\
\hline 1960 & 90,73 \\
1970 & 89,40 \\
1980 & 87,80 \\
1990 & 86,72 \\
2000 & 85,90 \\
2010 & 85,43 \\
2020 & 84,74 \\
\hline
\end{tabular}

FONTE: CENTRO LATINOAMERICANO DE DEMOGRAFIA. Boletin Demográfico: América Latina, projecciones de población 1950-2050. Santiago de Chile, v.27, n.54,1994.

Outros índices importantes para entender este processo de envelhecimento refere-se às taxas de: fecundidade, mortalidade geral e infantil, esperança de vida ao nascer e relação de dependência.

Taxa de fecundidade: esta taxa indica a distribuição de filhos por mulher em idade reprodutiva. Entre 1960 a 2020, seu comportamento foi o seguinte:

Tabela 5 - Taxa de Fecundidade Latino Americana de 1960 a 2020

\begin{tabular}{l|c}
\hline ANOS & T.FEC \\
\hline 1960 & 5.96 \\
1970 & 5,57 \\
1980 & 4,46 \\
1990 & 3,40 \\
2000 & 2,84 \\
2010 & 2,46 \\
2020 & 2,24 \\
\hline
\end{tabular}

FONTE: CENTRO LATINOAMERICANO DE DEMOGRAFIA. Boletin Demográfico: América Latina, projecciones de población 1950-2050. Santiago de Chile, v. 27, n. 54,1994.

Estud. interdiscip. envelhec., Porto Alegre, v.2, p.7-21, 1999. 
A Taxa Bruta de Mortalidade: indica a distribuição de mortes produzidas por cada 1000 (mil) indivíduos presentes em um momento e espaço determinado.

Tabela 6 - Taxa Bruta de Mortalidade em cada 1000 Habitantes.

\begin{tabular}{l|c}
\hline ANOS & T. B. MORT. \% o \\
\hline 1960 & 13,88 \\
1970 & 11.10 \\
1980 & 8,73 \\
1990 & 7,18 \\
2000 & 6,45 \\
2010 & 6,25 \\
2020 & 6.42 \\
\hline
\end{tabular}

FONTE: CENTRO LATINOAMERICANO DE DEMOGRAFIA. Boletin Demográfico: América Latina, projecciones de población 1950-2050. Santiago de Chile, v.27, n.54,1994.

Taxa de Mortalidade Infantil: refere-se ao número de óbitos entre crianças menores de 1 (um) ano por cada 1000 (mil) nascidas vivas em igual período.

Tabela 7 - Taxa de Mortalidade Infantil em cada 1000 Nascidos.

\begin{tabular}{l|c}
\hline ANOS & T. MORT. INF. \%o \\
\hline 1960 & 114,12 \\
1970 & 91,80 \\
1980 & 69,30 \\
1990 & 50,96 \\
2000 & 41,01 \\
2010 & 33,23 \\
2020 & 26,84 \\
\hline
\end{tabular}

FONTE: CENTRO LATINOAMERICANO DE DEMOGRAFIA. Boletin demográfico: América Latina, projecciones de población 1950-2050. Santiago de Chile, v.27, n. $54,1994$.

Estas taxas ao atuar em conjunto produzem alterações que incidem no aumento do índice de envelhecimento, já que uma queda na fecundidade (menor número de nascimentos) e na mortalidade (menor número de óbitos) produzem aumento na Esperança de Vida ao Nascer que é a media de anos que espera- 
se que viva um recém nascido, tendo em vista os níveis atuais da mortalidade.

Esta esperança de vida aumentou em forma dramática nas décadas que seguem a Segunda Guerra Mundial.

Assim a esperança de vida teve o seguinte comportamento:

Tabela 8 - Esperança de Vida ao Nascer, em Anos.

\begin{tabular}{l|c}
\hline ANOS & ESP. DE VIDA \\
\hline 1960 & 54,69 \\
1970 & 59,11 \\
1980 & 63,37 \\
1990 & 67,12 \\
2000 & 69.93 \\
2010 & 72,19 \\
2020 & 74,17 \\
\hline
\end{tabular}

FONTE: CENTRO LATINOAMERICANO DE DEMOGRAFIA. Boletin Demográfico: América Latina, projecciones de población 1950-2050. Santiago de Chile, v.27, n. 54,1994 .

\subsection{Consequiências do Envelhecimento Latino Americano}

O processo de envelhecimento na América Latina apresenta problemáticas a serem resolvidas com prioridade tanto na área econômica como social e política.

A relação entre a população em idade ativa (15 - 60 anos) e a população em idade inativa $(0$ - 14 e 60+) denomina-se relação de dependência, apresentando modificações alarmantes nas últimas décadas:

Tabela 9-Percentual de População Inativa em Relação a População Ativa.

\begin{tabular}{l|c}
\hline ANOS & R. DEP \% \\
\hline 1960 & 85.67 \\
1970 & 86,58 \\
1980 & 78,15 \\
1990 & 68,53 \\
2000 & 59,79 \\
2010 & 53,27 \\
2020 & 50,30 \\
\hline
\end{tabular}

FONTE: CENTRO LATINOAMERICANO DE DEMOGRAFIA. Boletin Demográfico: América Latina, projecciones de población 1950-2050. Santiago de Chile, v. 27, n. 54,1994.

Estud. interdiscip. envelhec., Porto Alegre, v.2, p.7-21, 1999. 
Isto significa que em 2020 existirão quase cinqüenta e uma (51) pessoas em idade de dependência por cada cem (100) pessoas em idade produtiva. Nas décadas anteriores este índice era maior devido a que a população de zero a catorze anos $(0$ - 14) era mais numerosa e a presença de idosos, sessenta anos e mais $(60+)$, era menor .

Tabela 10 - Percentual de Menores de 15Anos e de Maiores de 60 Anos.

\begin{tabular}{l|c|c}
\hline ANOS & $\mathbf{- 1 5}$ anos & $\mathbf{6 0}+\mathbf{a n o s}$ \\
\hline 1960 & 42,48 & 5,82 \\
1970 & 42,45 & 6,09 \\
1980 & 40,00 & 6,50 \\
1990 & 35,87 & 7,18 \\
2000 & 31,87 & 8,08 \\
2010 & 28,21 & 9,62 \\
2020 & 25,00 & 12,00 \\
\hline
\end{tabular}

FONTE: CENTRO LATINOAMERICANO DE DEMOGRAFIA. Boletin Demográfico: América Latina, projecciones de población 1950-2050. Santiago de Chile, v. 27, n. 54,1994.

Do ponto de vista dos custos sociais o envelhecimento populacional representa um grave peso para o Estado, que deverá esforçar-se para dar atendimento e satisfazer as necessidades de saúde, previdência, aposentadoria e condições com qualidade de vida a essa população.

\section{Brasil e o Processo de Envelhecimento}

O Brasil é um dos países da América Latina que tem experimentado um dos maiores aumentos em relação a sua proporção de idosos na população total. Isto significa uma acelerada tendência de envelhecimento populacional.

Nas ultimas décadas constata-se esta afirmação ao longo dos Censos e as projeções até 2020 a confirmam.

\subsection{População Idosa e Seu Crescimento}

A distribuição da população de sessenta anos e mais (60+) em números absolutos tem evoluído nas ultimas décadas na seguinte forma: 
Tabela 11 - Crescimento da População Brasileira com 60 Anos e mais, em Números Absolutos.

\begin{tabular}{l|c}
\hline ANOS & População 60 + anos \\
\hline 1960 & 3.330 .996 \\
1970 & 4.716 .208 \\
1980 & 7.216 .017 \\
1990 & 10.722 .705 \\
2000 & 13.090 .100 \\
2010 & 18.114 .300 \\
2020 & 27.173 .600 \\
\hline
\end{tabular}

FONTE: FUNDAÇÃO IBGE. Anuários Estatísticos, 1965, 1982, 1992, 1994 e 1996. Idem. Censo Demográfico Brasileiro. 1960, 1970, 1980 e 1991.

$O$ índice de envelhecimento populacional vem experimentando também uma tendência de aumento, que nas projeções para o século XXI mostram uma rápida elevação.

Tabela 12 - Índice de Envelhecimento em Percentuais.

\begin{tabular}{l|c}
\hline ANOS & Ind. Envelh.\% \\
\hline 1960 & 6.4 \\
1970 & 6.6 \\
1980 & 6.9 \\
1990 & 7.1 \\
2000 & 7.9 \\
2010 & 8.6 \\
2020 & 13.0 \\
\hline
\end{tabular}

FONTE: F UNDAÇÃO IBGE. Anuários Estatísticos, 1965, 1982, 1992, 1994 e 1996. Idem. Censo Demográfico Brasileiro. 1960, 1970, 1980 e 1991.

\section{2 Índices Demográficos Brasileiros e sua Participação no Envelhecimento Populacional.}

Com relação à distribuição por sexo da população idosa, a tendência brasileira é predominantemente feminina. Com o crescimento do número de pessoas nas faixas etárias mais avançadas, observa-se uma redução no percentual de homens nessa população, em números absolutos e relativos. 
Tabela 13 - População Idosa, em Números Absolutos e Relativos, Segundo o Sexo.

\begin{tabular}{c|c|c|c|c}
\hline ANO & Homens & \% & Mulheres & \% \\
\hline 1960 & $1,646,542$ & 50,00 & $1,665,878$ & 50,00 \\
1970 & $2,295,991$ & 49,00 & $2,420,217$ & 51,00 \\
1980 & $3,413,468$ & 47,00 & $3,805,549$ & 53,00 \\
1990 & $4,931,425$ & 46,00 & $5,791,280$ & 54,00 \\
2000 & $5,935,300$ & 45,00 & $8,164,800$ & 55,00 \\
2010 & $8,012,600$ & 44,00 & $10,101,700$ & 56,00 \\
2020 & $11,872,000$ & 44,00 & $15,301,600$ & 56,00 \\
\hline
\end{tabular}

FONTE: FUNDAÇÃO IBGE. Anuários Estatísticos, 1965, 1982, 1992, 1994 e 1996. Idem. Censo Demográfico Brasileiro. 1960, 1970, 1980 e 1991.

Entre os índices demográficos relacionados com o envelhecimento populacional estão as taxas de: fecundidade, mortalidade geral e infantil, esperança de vida ao nascer e relação de dependência.

Taxa de fecundidade: esta taxa tem experimentado profundas modificações através das décadas. A causa motivadora pode estar na inserção feminina significativa no mercado de trabalho assim como na utilização mais difundida de métodos anticonceptivos.

Tabela 14 - Taxa de Fedundidade

\begin{tabular}{l|c}
\hline ANOS & T.FEC \\
\hline 1960 & 6,21 \\
1970 & 5,76 \\
1980 & 4,01 \\
1990 & 2,50 \\
2000 & 2,04 \\
2010 & 1,85 \\
2020 & 1,81 \\
\hline
\end{tabular}

FONTE: FUNDAÇÃO IBGE. Anuários Estatísticos, 1965, 1982, 1992, 1994 e 1996. Idem. Censo Demográfico Brasileiro. 1960, 1970, 1980 e 1991.

Taxa Bruta de Mortalidade é número de óbitos por mil indivíduos em 16 
um determinado ano e região.

Tabela 15 - Taxa Bruta de Mortalidade em cada 1000 Habitantes.

\begin{tabular}{l|c}
\hline ANOS & T.B.Mort. \% o \\
\hline 1960 & 11,20 \\
1970 & 10,70 \\
1980 & 9,00 \\
1990 & 7,11 \\
2000 & 6,69 \\
2010 & 6,61 \\
2020 & 6,00 \\
\hline
\end{tabular}

FONTE: FUNDAÇÃO IBGE. Anuários Estatísticos, 1965, 1982, 1992, 1994 e 1996. Idem. Censo Demográfico Brasileiro. 1960, 1970, 1980 e 1991.

Taxa de Mortalidade Infantil: esta taxa determina o número de crianças que morrem, de cada mil que nascem, antes de completar o primeiro ano de vida. Ela é o índice demográfico que melhor reflete as condições de vida de uma população.

Tabela 16 - Taxa de Mortalidade Infantil em cada 1000 Nascimentos

\begin{tabular}{l|c}
\hline ANOS & T.Mort.Inf. \% o \\
\hline 1960 & 121,08 \\
1970 & 113,79 \\
1980 & 69,10 \\
1990 & 48,70 \\
2000 & 39,20 \\
2010 & 28,50 \\
2020 & 17,60 \\
\hline
\end{tabular}

FONTE: FUNDAÇÃO IBGE. Anuários Estatísticos, 1965, 1982, 1992, 1994 e 1996. Idem. Censo Demográfico Brasileiro. 1960, 1970, 1980 e 1991.

Como foi indicado anteriormente a ação em conjunto destas taxas produz modificações no comportamento demográfico da população, no que se refere a estrutura etária.

Estud. interdiscip. envelhec., Porto Alegre, v.2, p.7-21, 1999. 
Esperança de vida ao nascer: indica a média de anos que se espera que viva um recém nascido, tendo em vista os níveis atuais da mortalidade.

Tabela 17 - Esperança de Vida ao Nascer, em Anos.

\begin{tabular}{l|c}
\hline ANOS & Esp. De Vida \\
\hline 1960 & 51,64 \\
1970 & 52,67 \\
1980 & 61,76 \\
1990 & 65,90 \\
2000 & 68,51 \\
2010 & 71,77 \\
2020 & 75,51 \\
\hline
\end{tabular}

FONTE: FUNDAÇÃO IBGE. Anuários Estatísticos, 1965, 1982, 1992, 1994 e 1996. Idem. Censo Demográfico Brasileiro. 1960, 1970, 1980 e 1991.

\subsection{Conseqüência do Envelhecimento Brasileiro}

Um fato importante se refere a Relação de Dependência, que mostra a situação econômica que deverá ser observada, em relação a distribuição da população por idades ativas e passivas e o peso que suportará a população ativa.

Tabela 18 - Percentual da População Inativa em Relação à População Ativa.

\begin{tabular}{l|c}
\hline ANOS & R. DEP \% \\
\hline 1960 & 83,20 \\
1970 & 82,60 \\
1980 & 73,20 \\
1990 & 65,50 \\
2000 & 50,28 \\
2010 & 43,20 \\
2020 & 43,24 \\
\hline
\end{tabular}

FONTE: FUNDAÇÃO IBGE. Anuários Estatísticos, 1965, 1982, 1992, 1994 e 1996. Idem. Censo Demográfico Brasileiro. 1960, 1970, 1980 e 1991.

No ano 2020 existirão quase quarenta e quatro (44 ) pessoas em idade 
de dependência por cada cem (100) pessoas em idade produtiva. Com relação a diminuição do índice através das décadas, cabe a mesma explicação dada para a situação de América Latina.

Tabela 19 - Percentual de Menores de 15 Anos e de Maiores de 60

\begin{tabular}{l|c|c}
\hline ANOS & $\mathbf{- 1 5}$ & $\mathbf{6 0 + a n o s}$ \\
\hline 1960 & 43,00 & 4,70 \\
1970 & 42,01 & 5,06 \\
1980 & 38,20 & 5,10 \\
1990 & 34,72 & 7,30 \\
2000 & 28,30 & 7,90 \\
2010 & 23,61 & 9,83 \\
2020 & 21,40 & 13,60 \\
\hline
\end{tabular}

FONTE: FUNDAÇÃO IBGE. Anuários Estatísticos, 1965, 1982, 1992, 1994 e 1996. Idem. Censo Demográfico Brasileiro. 1960, 1970, 1980 e 1991.

Também é importante a razão de sexo na população idosa para observar a crescente feminização desta faixa etária. Este índice expressa o número de homens por cem (100) mulheres em um determinado grupo populacional.

Tabela 20 - Percentual de Homens Idosos em Relação à Mulheres Idosas.

\begin{tabular}{l|c}
\hline ANOS & R.de SEXO \% \\
\hline 1960 & 98,84 \\
1970 & 94,87 \\
1980 & 89,77 \\
1990 & 85,15 \\
2000 & 82,70 \\
2010 & 79,31 \\
2020 & 77,59 \\
\hline
\end{tabular}

FONTE: FUNDAÇÃO IBGE. Anuários Estatísticos, 1965, 1982, 1992, 1994 e 1996. Idem. Censo Demográfico Brasileiro. 1960, 1970, 1980 e 1991.

É necessário lembrar que, neste caso, o índice está indicando a relação considerando como único grupo a população de sessenta anos (60) e mais. 
Quando se faz por grupos qüinquienais ou decenais, este índice apresenta uma queda mais violenta.

Esta situação ajuda a fragilizar mais ainda os segmentos idosos dentro da população de um continente ou país, o que torna mais preocupante a perspectiva.

Será necessário investir em políticas sociais que permitam modificar e instaurar novas visões sobre este setor populacional.

\section{Conclusão}

O envelhecimento populacional tem um impacto importante na vida econômica, social e política de um continente ou país. Aumenta, como vimos, a razão de dependência, o que produz complicações econômicas à medida em que esta razão aumenta.

Segundo alguns economistas diminui a taxa de poupança já que os mais velhos vivem de suas economias acumuladas, especialmente em economias desenvolvidas.

As medidas de seguridade social, serviço de saúde e previdência também são afetadas exigindo do estado soluções rápidas.

A pobreza é outro problema da velhice, especialmente em países em vias de desenvolvimento onde os acelerados processos de urbanização e de industrialização aprofundam as mudanças sociais fundamentalmente neste mundo etário de 60 anos e mais que apresenta dificuldades de adaptação a novas situações.

Assim, os idosos constituem um desafio para a sociedade civil e para o Estado que devem procurar oferecer qualidade de vida apropriada e eficiente.

\section{Referências Bibliográficas}

1 ANZOLA, Elias; RESTREPO, Helena. Análisis comparativo del envejecimento en Brasil, Colombia, El Salvador, Jamaica y Venezuela. Cuaderno Técnico, Washington, n 38, 1994.

2 ASSOCIAÇÃO BRASILEIRA DE ESTUDOS POPULACIONAIS. Diversidades Brasileiras: um olhar demográfico. São Paulo 1996. 
3 CENTRO LATINOAMERICANO DE DEMOGRAFIA. Boletin Demográ-

fico: América Latina, projecciones de poblaciones 1950-2050. Santiago de Chile, v.27, n.54, 1994.

4 COALE, A The Demografic Transition. Proceding of the International Population Conference. Liege, IUSSP, vol. 1, 1973.

5 FUNDAÇÃO IBGE. Anuário Estatístico do Brasil. Rio de Janeiro, 1965, v.26.

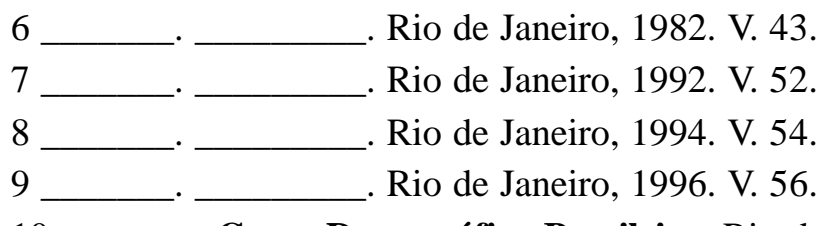

10

Censo Demográfico Brasileiro. Rio de Janeiro, 1960.

11 _. Rio de Janeiro, 1970.

12 . Rio de Janeiro, 1980.

13 _. Rio de Janeiro, 1991.

14 NATIONAL INSTITUTE ON AGING. Aging in the Americas into the XXI Century. Washington, D.C., E.N., 1998.

15 POPULATION ACTION INTERNATIONAL. Fondos para el futuro: como satisfacer la demanda de planificación familiar. Washington, D.C., E.E.N.N.1988.

16 POPULATION REFERENCE BUREAU. Datos y cálculos demográficos sobre los países y regiones del mundo. Washington, D.C., E.E.N.N.,1998.

17 POPULATION REFERENCE BUREAU. Las mujeres de nuestro mundo. Washington, D.C.,E.E.N.N.,1998.

18 RAMOS, Luiz Roberto. A Explosão Demográfica da Terceira Idade no Brasil: uma questão de saúde pública. Revista de Gerontologia, São Paulo, v. 1, n.1, p.3-8, mar. 1993.

19 VERAS, Renato P. Pais Jovens com Cabelos Brancos: a saúde do idoso no Brasil. Rio de Janeiro, Relume Dumará, UERJ, 1994. 